\title{
First average CsI neutron radius from the coherent elastic neutrino-nucleus scattering
}

\author{
Yu-Feng Li*i \\ Institute of High Energy Physics, Chinese Academy of Sciences, and School of Physical \\ Sciences, University of Chinese Academy of Sciences, Beijing 100049, China \\ E-mail: liyufeng@ihep.ac.cn
}

Using the first coherent elastic neutrino-nucleus scattering data, we determine the average neutron root-mean-square (rms) radius of ${ }^{133} \mathrm{Cs}$ and ${ }^{127} \mathrm{I}$. A model-independent value $R_{n}=5.5_{-1.1}^{+0.9} \mathrm{fm}$ is obtained using the symmetrized Fermi and Helm form factors. Meanwhile, the nuclear structure suppression of the full coherence is observed with a significance of $2.3 \sigma$.

ICHEP 2018, 39th International Conference on High Energy Physics

4-11 July 2018

Seoul, Korea

\footnotetext{
* Speaker.

$\dagger$ This work was supported in part by the National Natural Science Foundation of China under Grant No. 11835013, by the Strategic Priority Research Program of the Chinese Academy of Sciences under Grant No. XDA10010100, and by the CAS Center for Excellence in Particle Physics (CCEPP).
} 


\section{Introduction}

In analogy to the coherent behavior of electron-nucleus scattering, the coherent elastic neutrinonucleus scattering (CEvNS) was first predicted in 1974 by Freedman [1]. However, the first observation of CEvNS was just accomplished in 2017 by the COHERENT experiment [2] with a small sodium-doped CsI scintillator detector using a low-energy decay-at-rest neutrino beam from the Spallation Neutron Source at Oak Ridge National Laboratory.

When neutrino interacts with the nucleus via the exchange of the $Z$-boson, if the neutrino wavelength is much smaller that the nucleus size, the nucleon wave functions in the target nucleus are in phase with each other. Therefore, the nucleus will recoils as a whole and the interaction rates will be a coherent summation of each constituent nucleon. Moreover, the neutron coupling contribution is dominating among all the nucleons and the cross section is approximately proportional to the square of the neutron number. To be explicit, the differential CEvNS cross section for a nucleus with $Z$ protons and $N$ neutrons can be written as

$$
\frac{d \sigma_{v \mathrm{~N}}}{d T}(E, T) \simeq \frac{G_{\mathrm{F}}^{2} M}{4 \pi}\left(1-\frac{M T}{2 E^{2}}\right) \times\left[N F_{N}\left(q^{2}\right)-\varepsilon Z F_{Z}\left(q^{2}\right)\right]^{2}
$$

where $G_{\mathrm{F}}$ is the Fermi constant, $M$ is the nuclear mass, $F_{N}\left(q^{2}\right)$ and $F_{Z}\left(q^{2}\right)$ are the nuclear neutron and proton form factors respectively, and $\varepsilon=1-4 \sin ^{2} \vartheta_{\mathrm{W}} \simeq 0.0454 \pm 0.0003$, using the lowenergy PDG value of the weak mixing angle $\vartheta_{\mathrm{W}}[3]$.

The nuclear form factors $F_{N}\left(q^{2}\right)$ and $F_{Z}\left(q^{2}\right)$ are the Fourier transform of the corresponding neutron and proton charge distributions, which can be described using either the symmetrized Fermi (SF) form factor [4] or Helm form factor [5] with two independent parameters of the rootmean-square (rms) radius and the surface thickness. In our analysis, we take the parameters of the proton form factor from the muonic atom spectroscopy [6] with the $R_{p}^{\mathrm{Cs}}=\left\langle r_{p}^{2}\right\rangle_{\mathrm{Cs}}^{1 / 2}=4.804 \mathrm{fm}$ and $R_{p}^{\mathrm{I}}=\left\langle r_{p}^{2}\right\rangle_{\mathrm{I}}^{1 / 2}=4.749 \mathrm{fm}$. For the surface thickness, we assume a universal value of $0.9 \mathrm{fm}$ [7] for both the proton and neutrons. Thus we can use the CEvNS measurement to obtain the information of the neutron radius.

\section{Neutron radius}

In 2017, the COHERENT collaboration published the first observation [2] of CEvNS using the CsI scintillator detector and the low-energy decay-at-rest neutrino beam with a significance of 6.7 $\sigma$. The neutrino beam consists of a prompt component of monochromatic $v_{\mu}$ from stopped pion decays, and two delayed components of $\bar{v}_{\mu}$ and $v_{e}$ from the subsequent muon decays. The $14.6 \mathrm{~kg}$ CsI scintillator detector is located at a distance of $19.3 \mathrm{~m}$ from the neutrino beam source, with a linear relation between the observed number of photoelectrons $N_{\mathrm{PE}}$ and the nuclear kinetic recoil energy $T$ given by $N_{\mathrm{PE}}=1.17[T / \mathrm{keV}]$.

We fitted the COHERENT data in Fig. 3A of Ref. [2] with the least-squares function defined in Ref. [8] in order to get information on the value of the neutron rms radius $R_{n}$. The systematic uncertainties for the signal and background are $28 \%$ and $25 \%$ respectively. Both the SF and Helm form factors are used, and in both cases we obtained a minimum $\chi^{2}$ which is smaller than the $\chi^{2}$ 


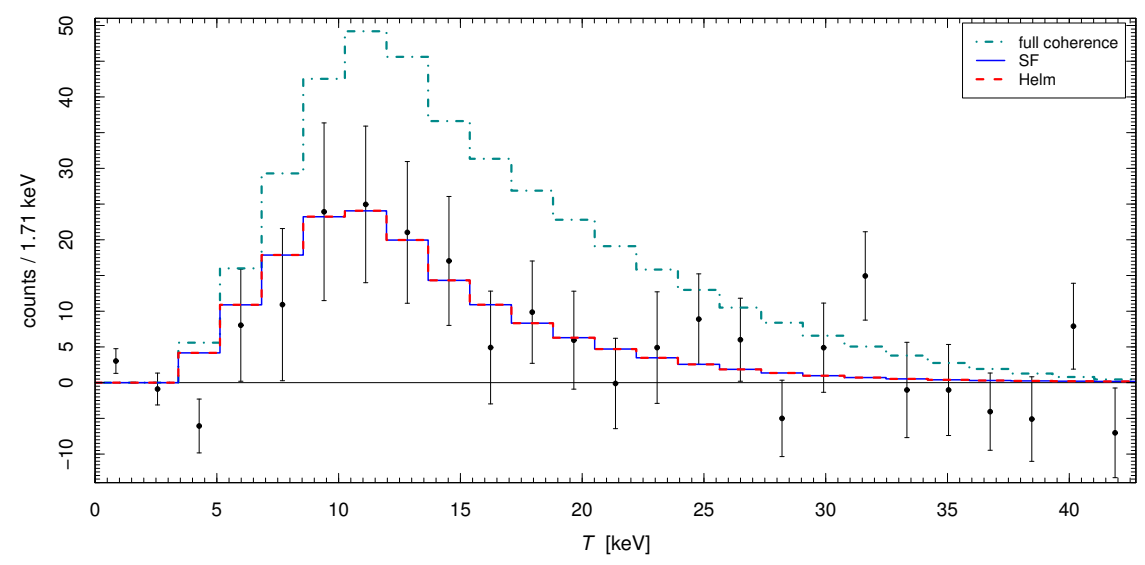

Figure 1: COHERENT data [2] versus the nuclear kinetic recoil energy $T$. The histograms represent the theoretical prediction in the case of full coherence (cyan dash-dotted) and the best fits obtained using the symmetrized Fermi (SF) distribution (blue solid) and Helm (red dashed) form factors.

corresponding to full coherence by 5.5. Hence, the hypothesis of full coherence has a $p$-value of $1.9 \%$ and there is a $2.3 \sigma$ evidence of the nuclear structure suppression of the coherence.

Figure 1 shows the best-fit results that we obtained using the symmetrized Fermi and Helm form factors. Figure 2 shows the corresponding marginal values of the $\chi^{2}$ as a function of $R_{n}$. One can see that the two parameterizations of the neutron form factor fit equally well the data and give practically the same result:

$$
R_{n}=5.5_{-1.1}^{+0.9} \mathrm{fm}
$$

This is the first determination of the neutron rms radius of a nucleus obtained with the neutrinonucleus scattering data. Note that it is practically model-independent, because it coincides for the SF and Helm form factors which correspond to reasonable descriptions of the nuclear density.

Future data of the COHERENT experiment may lead to a better determination of the neutron rms radius $R_{n}$. Keeping the systematic uncertainties and increasing ten times of the current number of protons on target, the data of the COHERENT experiment will allow us to determine $R_{n}$ within about $0.5 \mathrm{fm}$. In addition, if the systematic uncertainties are reduced by half or one quarter, $R_{n}$ can be determined within about 0.4 or $0.3 \mathrm{fm}$, respectively, which would be comparable to the measurement in the Parity-violating electron scattering experiments [9].

\section{Conclusion}

The measurement of the nuclear neutron density distribution is a topic of broad interest in the physics community. In particular, the corresponding rms radius $R_{n}$ and the difference between $R_{n}$ and the rms radius $R_{p}$ of the proton distribution are crucial ingredients of the nuclear matter Equation of State (EOS), which plays an essential role in understanding several processes, like nuclei in laboratory experiments, heavy ion collisions, and the structure and evolution of compact astrophysical objects as neutron stars [10, 11, 12,13,14].

In this work, we have determined for the first time the neutron rms radius of ${ }^{133} \mathrm{Cs}$ and ${ }^{127} \mathrm{I}$ from the data on coherent elastic neutrino-nucleus scattering in the COHERENT experiment. Taking into 


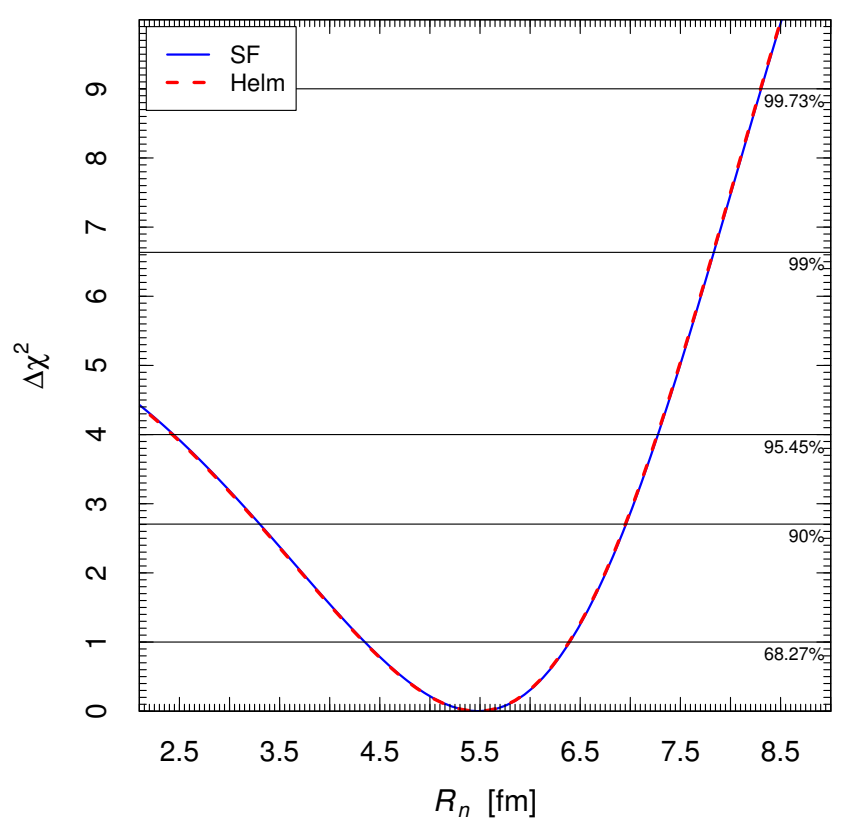

Figure 2: $\Delta \chi^{2}=\chi^{2}-\chi_{\min }^{2}$ as a function of the neutron rms radius $R_{n}$ obtained from the fit of the data of the COHERENT experiment [2] using the symmetrized Fermi (SF) and Helm form factors.

account the SF and Helm form factors, we obtained the model-independent value $R_{n}=5.5_{-1.1}^{+0.9} \mathrm{fm}$. We also found that the COHERENT data show a $2.3 \sigma$ evidence of the nuclear structure suppression of the full coherence.

\section{References}

[1] D. Z. Freedman, Phys. Rev. D 9, 1389 (1974).

[2] D. Akimov et al. [COHERENT Collaboration], Science 357, no. 6356, 1123 (2017).

[3] C. Patrignani et al. [Particle Data Group], Chin. Phys. C 40, no. 10, 100001 (2016).

[4] J. Piekarewicz, A. R. Linero, P. Giuliani and E. Chicken, Phys. Rev. C 94, no. 3, 034316 (2016).

[5] R. H. Helm, Phys. Rev. 104, 1466 (1956).

[6] G. Fricke, C. Bernhardt, K. Heilig, L. A. Schaller, L. Schellenberg, E. B. Shera and C. W. de Jager, Atom. Data Nucl. Data Tabl. 60, 177 (1995).

[7] J. Friedrich and N. Voegler, Nucl. Phys. A 373, 192 (1982).

[8] M. Cadeddu, C. Giunti, Y. F. Li and Y. Y. Zhang, Phys. Rev. Lett. 120, no. 7, 072501 (2018).

[9] S. Abrahamyan et al., Phys. Rev. Lett. 108, 112502 (2012).

[10] B. A. Brown, Phys. Rev. Lett. 85, 5296 (2000).

[11] C. J. Horowitz and J. Piekarewicz, Phys. Rev. Lett. 86, 5647 (2001).

[12] P.-G. Reinhard and W. Nazarewicz, Phys. Rev. C 81, 051303 (2010).

[13] M. B. Tsang et al., Phys. Rev. C 86, 015803 (2012).

[14] G. Hagen et al., Nature Phys. 12, no. 2, 186 (2015). 